\title{
Beneficios Económicos de la Gestión de la Demanda y la Energía Autogenerada en el Contexto de la Regulación Colombiana
}

\author{
Álvaro R. Restrepo ${ }^{1}$, Sandra E. Nope ${ }^{2}$, Delio E. Enríquez ${ }^{1}$ \\ (1) Fac. de Ingeniería de Electrónica y Telecomunicaciones, Univ. del Cauca, Calle 5 No. 4-70, Popayán- \\ Colombia. (e-mail: arestrepo@unicauca.edu.co; delio@unicauca.edu.co) \\ (2) Escuela de Ingeniería Eléctrica y Electrónica, Universidad del Valle, Calle 13 No. 100-00, Cali - \\ Colombia. (e-mail: sandra.nope@correounivalle.edu.co)
}

Recibido Jun. 13, 2017; Aceptado Ago. 18, 2017; Versión final Sep. 12, 2017, Publicado Feb. 2018

\begin{abstract}
Resumen
En este trabajo se determinan los beneficios económicos del funcionamiento de un sistema de autogeneración eléctrica con tecnologías de energías renovables. El sistema está conectado a la red tradicional $\mathrm{y}$, tiene las opciones de participar en programas de respuesta a la demanda y exportar excedentes de generación. Las variables del modelo de gestión, fueron identificadas a partir de las leyes y lineamientos establecidos por las entidades del sector eléctrico colombiano. Se demuestra que el modelo puede ser aplicado a empresas de diferentes sectores económicos y con diferentes curvas de carga. La incertidumbre de la generación y del cálculo de los beneficios se aborda mediante Simulación de Monte Carlo. Se presenta un caso de estudio en el que, utilizando un sistema de generación fotovoltaico se aplica el modelo propuesto a los sectores industriales y de servicios. Esto permite analizar los beneficios que pueden tener los inversionistas variando su capacidad de energía autogenerada.
\end{abstract}

Palabras clave: autogeneración, respuesta a la demanda, incertidumbre, energías renovables, beneficios económicos

\section{Economic Benefits of Demand Management and Self- Generated energy in the Context of Colombian Regulations}

\begin{abstract}
In this paper the economic benefits of the operation of an electric self-generation system with renewable energy sources are determined. The system is connected to the traditional grid and, it has the options to participating in the programs of demand response and to export its surpluses of generation. The variables of the management model were identified based on the Colombians laws and guidelines, established by the government entities of the electricity sector. The results show that the model can be applied to companies from different economic sectors using different curves of load. The uncertainty of the generation and the calculation of the profits were approached with Monte Carlo Simulation. A case of study is presented where a photovoltaic generation system was included, and the proposed model was applied to the industrial and service sectors. This allows analyzing the different benefits that investors can receive varying the capacity of self-generation.
\end{abstract}

Keywords: self-generation, demand response, uncertainty, renewable energy, economic benefits 


\section{INTRODUCCIÓN}

Uno de los inconvenientes de la aplicación de las políticas energéticas encaminadas al fomento de proyectos de autogeneración empleando las Fuentes de Energía Renovables (FER), es la identificación de los beneficios económicos que pueden llegar a obtener los inversionistas. Teniendo en cuenta la perspectiva tecno-económica, un aspecto crítico es determinar cómo los autogeneradores pueden hacer un control eficiente de los recursos, y a la vez, obtener beneficios económicos en el corto y largo plazo; considerando los incentivos que promueven los gobiernos a las inversiones en FER, la incertidumbre de este tipo de proyectos y, la posibilidad de participar en Programas de Respuesta a la Demanda (PRD). El Plan de Acción Nacional sobre respuesta a la demanda del Departamento de Energía de Estados Unidos (DOE, en inglés) define los PRD, como: la capacidad que tienen los consumidores para reducir su consumo de energía ante diferentes ofrecimientos, ya sea, a una señal de precios o de confiabilidad de su operador del sistema, u otros agentes del mercado eléctrico (DOE, 2010). Los PRD facilitan ahorros en los costos y el desplaza-miento temporal de las cargas pico, arbitrando entre períodos de baja y alta generación (Zhou et al., 2015).

De esta manera, las compañías y los clientes pueden beneficiarse de los Modelos de Gestión de la Demanda (MGD), que pueden ayudar a la operación de los mercados de electricidad, de forma que se reduzcan los picos de la demanda y la volatilidad de los precios (Mohsenian-Rad et al., 2010). Según Dong et al. (2016) los MGD, además de optimizar la interacción con los usuarios, proveen múltiples servicios, incrementan la eficiencia del uso de la energía, podrían optimizar la confiabilidad del sistema y, fomentar el consumo de las FER. De acuerdo con Wang et al. (2013), los dos principales beneficios del uso de los PRD, son: la reducción de los picos de la demanda y la satisfacción de la demanda a través del uso de renovables. Zhang et al. (2017) consideran que la gestión de la demanda tiene múltiples beneficios: la gestión de la carga de los consumidores, la reducción de la necesidad de nueva infraestructura de generación, transmisión y distribución, así como ahorros en inversiones, y contribuir a la estabilidad y confiabilidad de los sistemas de potencia. En este trabajo se trata a los PRD como parte de los MGD, ya que los PRD son una estrategia de los operadores de la red, mientras que los MGD son integrales y se abordan desde la perspectiva del consumidor eléctrico, quien puede hacer más eficiente su consumo con o sin PRD.

El análisis de los incentivos brindados a las inversiones en FER, ha sido abordado en otros trabajos: en Zhang, et al. (2014), valoran las tecnologías fotovoltaicas (FV) utilizando la Teoría de Opciones Reales (TOR), en el marco de las políticas del gobierno Chino. El impacto de las tarifas eléctricas definidas en el esquema europeo y la conveniencia de invertir en FER, es evaluado por Ritzenhofen y Spinler (2016), utilizando TOR. En Bahrami y Sheikhi (2015), realizan el modelamiento de los beneficios de un sistema eólico, en el marco de las políticas tarifarias de Australia. En Alemania, los incentivos a los mercados premium de generación con FER, son evaluados por Genoese et al. (2016), realizando la modelación económica de los beneficios establecidos para las tecnologías eólicas. Las opciones de un autogenerador en Colombia, fueron modeladas por Restrepo et al. (2016a), utilizando técnicas financieras y de inteligencia artificial. En Zhang et al. (2017), se revisan y analizan los mecanismos y restricciones para la implementación de PRD, en el marco de las reformas propuestas por el gobierno Chino en el 2015. Los modelos de gestión de la demanda que utilizan como base los PRD, comúnmente se han formulado desde el punto de vista de la empresa prestadora del servicio, por ejemplo: en Aalami et al. (2015), analizaron los beneficios que pueden obtener los clientes de una empresa que están conectados a una red tradicional, a través de un modelo lineal y no-lineal de tiempo, con un enfoque en el método PRD Momento de Uso (TOU, por sus siglas en inglés) y, en Abdi et al. (2015), los beneficios de un distribuidor son maximizados a través de un PRD de incentivos, un PRD de emergencia y control de carga directa.

De acuerdo con lo anterior, los sistemas de generación con base en FER y que incluyan PRD, deben ser evaluados en el marco de la normas del lugar en que se implementen. A diferencia de trabajos previos, en este artículo se determinan los beneficios de la operación de un sistema de autogeneración, a partir de un MGD planteado en función de: la demanda, la autogeneración, el precio de la electricidad y, la elasticidad de la demanda, y teniendo en cuenta los lineamientos establecidos en las leyes colombianas, a partir de los cuales se determinó las posibilidad de gestionar los flujos de potencia para incrementar los beneficios económicos a través de un PRD y/o venta de excedentes de potencia. EI MGD planteado, permite determinar tres tipos de beneficios económicos, a los que podrían acceder los autogeneradores en un día de operación del sistema. Se plantean las ecuaciones para el cálculo de los beneficios, considerando: la sustitución de compras a la red tradicional, la exportación de excedentes y, un PRD que se basa en el método Momento de Uso (TOU, por su sigla en inglés). La incertidumbre de las FER y del cálculo de los beneficios se simula con Monte Carlo. A través de un caso de estudio en el sector comercial e industrial, se determinan los beneficios económicos a los que puede acceder un autogenerador con la implementación de proyectos FV en el marco de la regulación colombiana. 


\section{ANTECEDENTES DE LA REGLAMENTACIÓN COLOMBIANA}

Las leyes 142 y 143 de 1994, reglamentan los servicios públicos de energía en Colombia. El Artículo 11 de la Ley 143, introdujo el concepto de autogenerador. La Resolución 084 de 1.996, de la Comisión de Regulación de Energía y Gas (CREG), definió la forma en la cual se llevaría a cabo esta actividad. En la década del 2000, se promulgaron normas para promover el desarrollo de las FER: la Ley 629 del 2000 aprobó el Protocolo de Kyoto; la Ley 697 de 2001, estableció el marco normativo para el uso racional y eficiente de la energía y, promueve, la utilización de fuentes no convencionales de energía; y, en el año 2002, para dar un impulso a los proyectos de generación con FER, se establece una excepción a la renta por un período de quince años a las ventas de energía.

En el año 2006, mediante la Resolución CREG-071, se establece la Demanda Desconectable Voluntaria, mecanismo que respalda las obligaciones de energía firme de las unidades de generación que participan en el Cargo por Confiabilidad. Mediante Resolución CREG-063 de 2010, se adopta este esquema para la Demanda Desconectable Voluntaria, como la cantidad de consumo de energía eléctrica diaria (kWH/día). Ésta, se realiza mediante un cambio de precio que obliga al Generador a pagar al Comercializador, mediante un pacto libre, en una relación contractual bilateral entre ellos (el Comercializador representa a los usuarios interesados). En esta Resolución, se excluye la participación de los autogeneradores (según lo define el artículo 11 de la ley 143 de 1994).La Resolución CREG-203 de 2013, establece: todo usuario que haga parte de la Demanda Desconectable Voluntaria, deberá permitir la interrogación de la información remota y de los parámetros del medidor. La Resolución 063, no explicita los PRD; sin embargo, objetivo y características son similares a los PRD por Programas de recompra.

EI "ESTATUTO DE LA AGENCIA INTERNACIONAL DE ENERGÍAS RENOVABLES (IRENA)", fue acogido en Colombia mediante la Ley 1665 de 2013, y para dar viabilidad a éste, el 21 de mayo de 2014 se promulga la ley 1715 (L1715), la cual regula la integración de las energías renovables no convencionales, al Sistema Interconectado Nacional; se introduce y definen normas para la implementación de PRD. El Artículo 5, establece definiciones que sirven de referencia para el presente trabajo, entre otras: medición bidireccional, excedentes de energía (producto de la actividad de autogeneración) y PRD. Los mecanismos para implementación de los PRD, fueron establecidos en el Decreto 2492 de diciembre de 2014, y contempla: 1) política energética en materia de entrega de excedentes de autogeneración; 2) concepto de autogeneración y la define como: actividad realizada por personas naturales y/o jurídicas para atender sus propias necesidades, que pueden ser entregadas a la red, previa formalización de un contrato de respaldo con el operador de red o transportador al cual se conecten; 3) objetivo de los PRD: lograr no sólo la confiabilidad en el Sistema Interconectado Nacional, sino también respaldar Obligaciones de Energía Firme, reducir los precios en la Bolsa de Energía y los costos de restricciones; 4) "incluir en el diseño de los cargos que remuneran las actividades de transmisión y distribución, tarifas horarias y/o canasta de tarifas de forma tal que permitan incentivar económicamente el uso más eficiente de la infraestructura y la reducción de costos de prestación del servicio". Según el Documento CREG-097, los autogeneradores al no tener las características de un generador, deberían ser representados por agentes del mercado (generadores o comercializadores), para llevar a cabo la entrega de excedentes al sistema y, el precio que les será reconocido por la venta, dependerá de las condiciones establecidas con el agente que lo represente en el mercado mayorista (CREG, 2014a). En la Resolución No 281 del 2015, la Unidad de Planeación Minero Energética, definió en un (1) MW el límite máximo de potencia de autogeneración, que debe corresponder a la capacidad instalada del sistema de generación.

\section{MODELO DE GESTIÓN DE LA DEMANDA PARA AUTOGENERADORES}

Teniendo en cuenta las normas vigentes, para un autogenerador que esté dispuesto a participar en un PRD y requiera elaborar, tanto un plan de gestión de compras como de generación de su sistema, se propone el modelo de gestión de demanda (MGD) que se basa en la ecuación (1).

$M G D=f\left(G, P, D^{*} \varepsilon_{p}\right)$

Donde $\mathrm{G}$ es la generación del sistema FER; $\mathrm{P}$ es el precio de la electricidad en el mercado; $\mathrm{D}$ es la demanda de potencia del autogenerador y, $\varepsilon_{p}$ es el factor de elasticidad de la demanda frente al precio en el sector empresarial. La G en el caso de las FER, está determinada por la disponibilidad de la fuente (sol, viento, biomasa, etc.) y por su comportamiento estocástico.

Los precios de la electricidad en los mercados competitivos como el colombiano, se clasifican generalmente en dos tipos: precios de la energía transada en bolsa (PB) y precios de contratos a largo plazo (PC); a través 
de los PC, se busca reducir la incertidumbre manifiesta de los PB (Povh et al., 2010). Se supone que estos contratos con distintas fechas y distintos períodos de vencimiento, reflejan las expectativas reales del comportamiento de los precios futuros. En los mercados eléctricos liberalizados, el precio se caracteriza por su alta volatilidad (Muñoz et al., 2010), es decir, es fluctuante.

Un aspecto importante que se debe considerar en la formulación de los MGD, es el Índice de Elasticidad de la Demanda $\left(\varepsilon_{p}\right)$, utilizado para describir la respuesta al consumo de la energía eléctrica frente a las señales de precio del mercado de electricidad (Hopper et al., 2006). Estas conclusiones fueron obtenidas, cuando llevaron a cabo una comparación de las elasticidades de los diferentes sectores económicos en Estados Unidos y encontraron que el sector industrial presentaba una mejor respuesta a los cambios en la energía eléctrica. Los siguientes son los principales beneficios ecónomicos que se considera, puede obtener un autogenerador de acuerdo con los lineamientos normativos de Colombia mencionados arriba: beneficios por sustitución de compras a la red tradicional con autogeneración $\left(B_{s}\right)$; beneficios por exportación ( $\left.B_{e}\right)$, y beneficios por participar en programas de respuesta a la demanda ( $\left.B_{R D}\right)$

\section{Beneficios por sustitución de compras a la red tradicional con energía autogenerada $\left(B_{s}\right)$}

Se asume que el autogenerador hace uso en todo momento de la producción de potencia que esté disponible en el sistema. Los $B_{s}$ son independientes de los costos nivelados de la electricidad, ya que los costos de la inversión inicial en tecnologías FER, son considerados como un costo hundido y, los costos de operación y mantenimiento por kWh en Colombia para las FV, son inferiores al precio del kWh (Restrepo et al. 2016b), que paga el consumidor; además, en este trabajo se calculan los beneficios de la operación y no la viabilidad de la implementación de un proyecto de autogeneración. La misma consideración se aplica en los otros tipos de beneficio.

Si los cálculos se realizan con una tarifa constante (sin incluir PRD), los Bs para un período de 24 horas, se calculan con la ecuación (2).

$\widetilde{\mathrm{B}}_{\mathrm{s}}=\sum_{1}^{24} \operatorname{Min}\left(\widetilde{\mathrm{AG}}_{\mathrm{T}} ; \widetilde{\mathrm{D}}_{\mathrm{T}}\right) \star \widetilde{\mathrm{PCl}}$

Donde AGT es la autogeneración total promedio; DT es el promedio de la demanda total; PCl, es el precio de compra promedio del consumidor industrial, calculado con base en las tarifas históricas del comercializador. En este trabajo el símbolo de énfasis $(\sim)$ que se coloca sobre las variables, indica que es el valor promedio, como resultado de un proceso de simulación.

\section{Beneficios por exportación $\left(B_{e}\right)$}

Son el resultado de la venta de los excedentes de autogeneración a la red de servicios. De acuerdo con el Decreto 2469, la autogeneración no puede ser mayor al $100 \%$ de la demanda promedio, y los autogeneradores a gran escala, están obligados a suscribir contratos de respaldo con el operador de la red (o transportador) al cual se conecten. Para las transacciones comerciales, deberán ser representados ante el mercado mayorista por un agente comercializador o por un agente generador. Lo anterior supone que el precio del kWh será igual al negociado en el mercado mayorista. Los beneficios por venta de excedentes en un día se calculan de acuerdo con las ecuaciones (3) y (4).

$\widetilde{\mathrm{B}}_{\mathrm{e}}=\sum_{1}^{24} \operatorname{Max}\left[\left(\widetilde{\mathrm{AG}}_{\mathrm{T}}-\mathrm{D}_{\mathrm{T}}\right) \tilde{\mathrm{I}}_{\mathrm{ve}} ; 0\right]$

$\tilde{I}_{v e}=\widetilde{P B}_{i}^{*}\left(1-G_{c}\right)-C_{t}$

Donde $\mathrm{B}_{\mathrm{e}}$ son los ingresos promedio por concepto de venta de excedentes; lve es el ingreso neto por la venta de cada kWh; $\mathrm{PB}_{i}$ es el precio promedio de la electricidad en bolsa para el período de cálculo; $\mathrm{G}_{c}$ porcentaje de gastos contractuales derivados de la representación del comercializador y, $\mathrm{C}_{\mathrm{t}}$ cargo por uso del sistema por transmisión del kWh.

De acuerdo con lo anterior, los beneficios de un autogenerador que no participe en PRD, serían: $\widetilde{B}_{S}$, cuando DT>AGt; o $B_{s}+B_{\text {e }}$ sí $D_{\text {T }} \leq A G$ T. 


\section{Beneficios por participar en Programas de Respuesta a la Demanda $\left(B_{R D}\right)$}

Cuando los clientes participan en PRD, hay tres formas en los que ellos pueden modificar su consumo eléctrico (Siano, 2014): 1) reduciendo su consumo mediante estrategias de reducción de la carga; 2) desplazando el consumo a diferentes períodos de tiempo y, 3) usando autogeneración de energía, así limita su dependencia de la red principal. A diferencia de los MGD tradicionales, en este trabajo, los anteriores planteamientos son considerados como una estrategia de gestión, que tiene el inversionista sobre la administración de los flujos de potencia (demanda de la red y autogenerada) y de caja. La implementación de los $P R D$, requieren de la definición de lo que se ha denominado Demanda Pico $\left(D_{p}\right)$, que describe un período o períodos, en el cual, la potencia eléctrica requerida por el consumidor es más alta que el promedio de la demanda. Con base en la $D_{p}$, se realizan las proyecciones y capacidad de los sistemas de generación a largo plazo. Otro elemento de implementación, es la denominada Demanda Valle ( $\left.D_{v}\right)$, que es el período en el que la demanda de potencia es baja (EIA, 2016).

Por cuanto la reglamentación de los PRD en Colombia es relativamente reciente, hay aspectos que no han sido claros sobre cómo serán aplicados; por lo anterior, se plantean los siguientes supuestos para el cálculo de los beneficios derivados de los PRD: 1) el autogenerador participa en un PRD-TOU con dos períodos de consumo demanda pico y demanda valle, que se fijan con base en la demanda promedio que es obtenida a partir de los valores históricos de la demanda de potencia; además, para cada período de demanda, se fija una tarifa pico $\left(T_{p}\right)$ y una tarifa valle $\left(T_{v}\right)$, siendo $\left.T_{p}>T_{v} ; 2\right)$ el autogenerador tiene libertad de utilizar la producción de potencia de su sistema en cualquier período de tiempo; 3) en condiciones de escasez se pueden conseguir grandes incentivos por medio de los PRD para un autogenerador; esto en concordancia con la propuesta presentada por CREG (documento CREG-056), donde "se propone un esquema similar a la demanda desconectable voluntaria (DDV), como un mecanismo de RD, en condiciones de escasez, pero con la diferencia que es la demanda la que reduce su consumo en respuesta a la señal de precio del mercado mayorista" (CREG, 2014b). La propuesta establece unos lineamientos para los usuarios, quienes están representados por un comercializador que tiene la misión de reducir la demanda en condiciones de escasez, la cual, puede ser ofertada al mercado diario con el propósito de reducir energía, cuando el precio de bolsa del pre despacho ideal, sea mayor al precio de oferta de reducción; 4) para el mercado eléctrico en Colombia y, de acuerdo con información de ISAGEN (2005), los períodos de $D_{p}$ se encuentran entre las 09:00 a 12:00 y 18:00 a 21:00 horas; 5) el valor del kWh en los PRD, se calcula con base en el documento CREG-056, en el cual se propone un $108 \%$ del precio de escasez; 6 ) la cantidad de potencia ahorrada en un PRD, se calcula con base en el índice de elasticidad del sector productivo en que se encuentran los consumidores. Este ahorro, no es más que el porcentaje de consumo de potencia que es posible disminuir o desplazar [ver ecuación (5)], durante las horas de mayor consumo. Los beneficios por ahorro se calculan de acuerdo con los incentivos sugeridos para un PRD por la CREG [ver ecuación (6)].

$$
\begin{aligned}
& Q_{i}=\varepsilon_{p}{ }^{*} D_{p} \\
& B_{A}=I_{R D}{ }^{*} Q_{i}
\end{aligned}
$$

Donde $Q_{i}$ es la cantidad de potencia ahorrada en el período de cálculo; $\varepsilon_{p}$ es el índice de elasticidad de la demanda del sector empresarial; $D_{p}$ es la demanda de potencia en el período definido como pico; $B_{A}$ son los beneficios económicos por participar en PRD e, IRD incentivo económico por cada kWh dejado de consumir.

Cuando el consumidor cuenta con sistemas de autogeneración de electricidad y participa en PRD-TOU, además de los ingresos por disminución de su consumo, tiene la opción de cubrir el total o parte de la $D_{p}$ con potencia autogeneración en ese período (o en otro período, si cuenta con sistemas de almacenamiento de potencia). En este caso, para el cálculo de los $B_{\mathrm{RD}}$ durante el período pico (BRD), se debe tener presente si la autogeneración es mayor, menor o, igual a $D_{p}$ descontado el $\varepsilon_{p}$ [ver ecuación (7)], ya que sólo se reconocerán ingresos ( $\mathrm{I}_{\mathrm{RD}}$ ) para la cantidad de potencia que se encuentra por encima de la demanda (los excedentes son exportados a la red).

$$
\left.\widetilde{\mathrm{B}}_{\mathrm{RDp}}=\sum_{1}^{24} \mathrm{~B}_{\mathrm{A}}+\operatorname{Min}\left[\widetilde{\mathrm{AG}}_{\mathrm{p}}, \mathrm{D}_{\mathrm{p}}{ }^{*}\left(1-\varepsilon_{\mathrm{p}}\right)\right]^{*} \mathrm{~T}_{\mathrm{p}}\right]
$$

En el caso de la demanda valle ( $\left.D_{v}\right)$ los beneficios con PRD (BRDp) son similares al cálculo del $B_{s}$ [ver ecuación (2)]; a diferencia de éste, en lugar de $\mathrm{PCl}$, se considera la tarifa valle ( $\mathrm{T} v$ ), y se calculan con la ecuación (8):

$\widetilde{\mathrm{B}}_{\mathrm{RDv}}=\sum_{1}^{24} \operatorname{Min}\left(\widetilde{\mathrm{AG}}_{\mathrm{v}} ; \widetilde{\mathrm{D}}_{\mathrm{v}}\right)^{*} \mathrm{~T}_{\mathrm{v}}$ 


\section{CASO DE ESTUDIO}

El caso de estudio, se desarrolla para dos tipos de empresas: una comercial dedicada a las ventas al retail y otra agro-industrial que produce aceite de palma, pero con diferentes curvas de carga y temporalidad de la demanda pico. Para autogeneración se supuso paneles FV, ya que, de acuerdo con los índices de radiación en Colombia, éstas son propicias para la generación (UPME e IDEAM, 2005). En las dos empresas se realizaron los cálculos de los beneficios para un día promedio de consumo de electricidad, con base en la demanda del año 2015. Estos datos se obtuvieron de la empresa proveedora del servicio eléctrico de la región en que se encuentran ubicadas. La industria presentó una demanda total promedio diaria de $\mathrm{D}_{\mathrm{T}}=2.873,59$ kWh y, el hipermercado, una $D_{T}=3.548,71$. Se consideraron dos tipos de escenarios: Esc. 1 , se utilizaron 300 paneles de $260 \mathrm{Wp}$, y en el Esc. 2, 1500. En las Figuras 1, 2, 3 y, 4 se presentan las curvas de demanda por hora y promedio y, la generación FV promedio por hora, de la industria y del hipermercado.

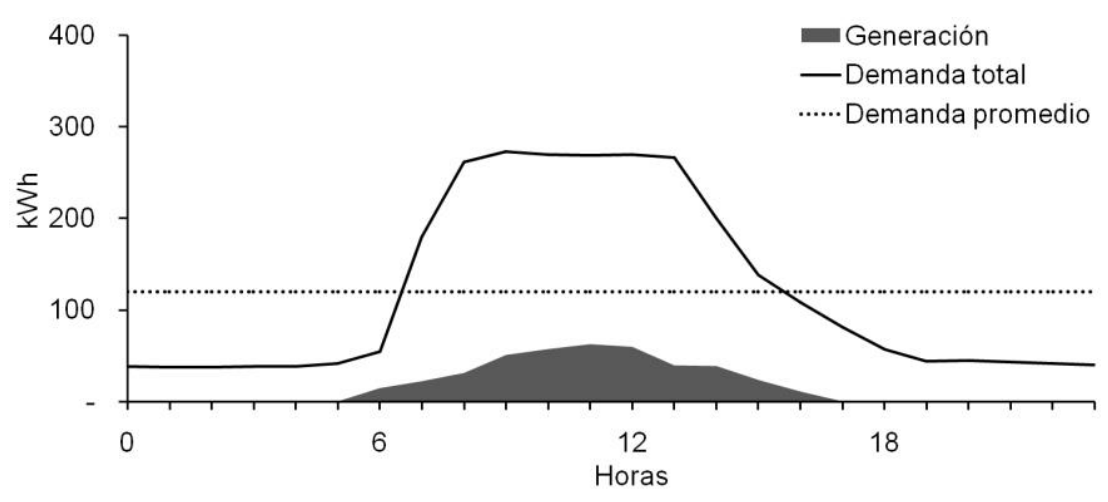

Fig. 1: Empresa industrial, Escenario1

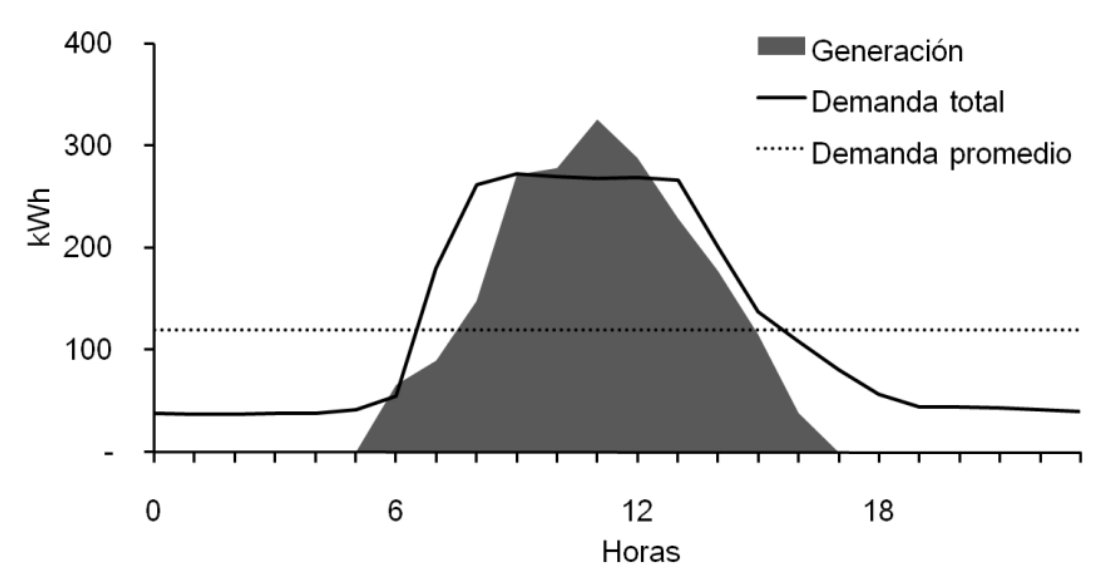

Fig. 2: Empresa industrial, Escenario 2

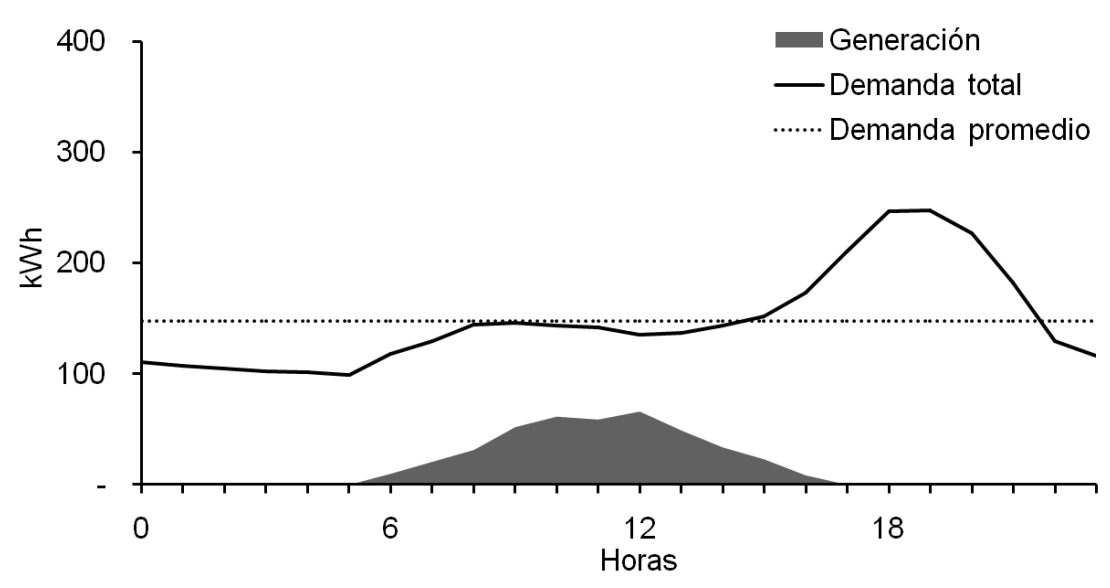

Fig. 3: Hipermercado, Escenario 1 


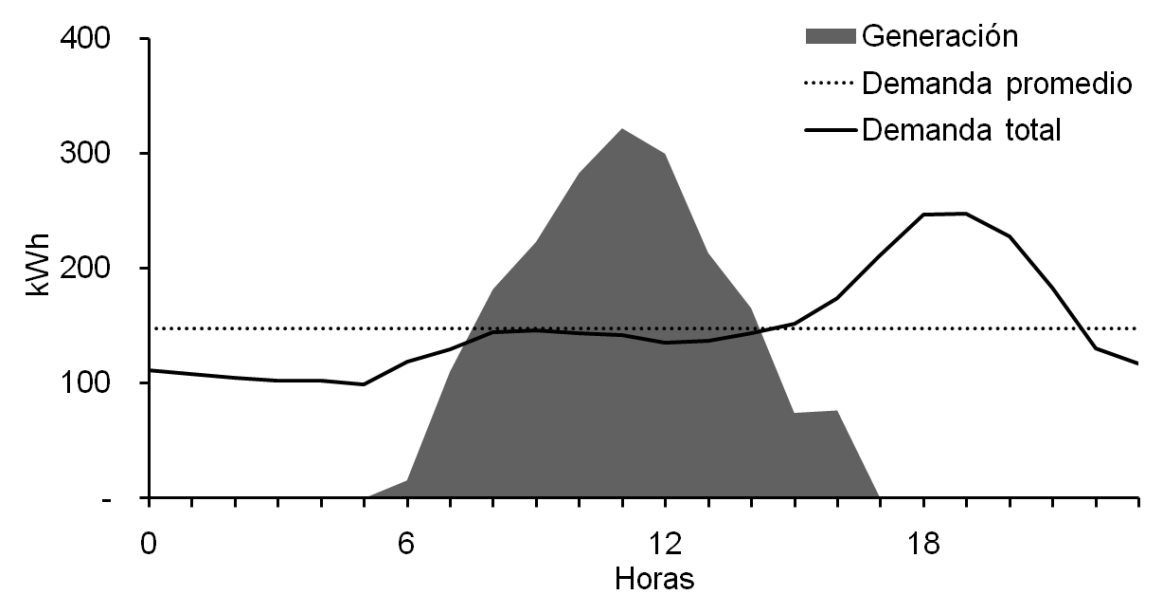

Fig. 4: Hipermercado, Escenario 2

Las entradas de las tecnologías que permiten convertir una fuente natural como la radiación solar y el viento en electricidad, son series que tienen un comportamiento estocástico a lo largo del día y el año. Para obtener el promedio de generación, a partir de los datos obtenidos de la NASA (2015), se realizó un proceso de Simulación de Monte Carlo, mediante una función de distribución normal y cuya media y desviación estándar para la radiación solar, fueron: Esc. $1=4,68 \mathrm{kWh} / \mathrm{m}^{2} \mathrm{y}$, Esc. $2=0,23 \mathrm{kWh} / \mathrm{m}^{2}$. Se puede apreciar la diferencia en la temporalidad de las curvas de carga entre las dos empresas. Es claro que la generación de electricidad a partir de FV, en el caso del ejemplo de la empresa industrial, generará mayor impacto sobre los beneficios en el período pico, frente al caso del hipermercado, en el que su período pico se presenta en el período nocturno.

Para el cálculo de los beneficios por sustitución, exportación y PRD, son requeridos los diferentes tipos de precios de la electricidad en el mercado: precios en la bolsa (PB) al por mayor; los precios de compra de los consumidores industriales y comerciales (PCl) y, los precios de escasez (PE). Estos se obtuvieron de la base de datos del Sistema de Información Eléctrico Colombiano (UPME, 2016). Para el cálculo de los beneficios, se tomó el promedio de los precios históricos correspondiente al año 2015, que fueron: $\mathrm{PB}=228,87$ $\$ C O P / k W h ; P C l=381,16 \$ C O P / k W h$ y, $\mathrm{PE}=330,18 \$ C O P / k W h$. Para el cálculo de los beneficios por exportación de excedentes, además del PB de la electricidad, se consideran los cargos por uso del Sistema de Transmisión Regional $\left(\mathrm{C}_{t}\right)$. En el año 2015, el promedio diario del $\mathrm{C}_{t}$ fue de 17,8 \$/kWh (XM S.A. E.S.P., 2015). Para los gastos contractuales $\left(G_{c}\right)$, se supuso un valor del $5 \%$ del $P B$ por cada $k W h$ exportado a la red.

Las tarifas supuestas para determinar los $\mathrm{B}_{\mathrm{RD}}$ se establecieron con base en el PCl. Se estimó un incremento del 8\% (valor igual al sugerido por la CREG para PRD) para la $T_{p}$, y un decremento por el mismo valor, para $T_{v}$. La cantidad de potencia ahorrada $\left(Q_{i}\right)$, la cual es calculada con base en el índice de elasticidad de la demanda, se determinó con base en el estudio desarrollado por Baratto-Callejas (2010) en Colombia, donde se encontró que, la elasticidad del sector agro-industrial, es del 0,16 y la del comercio del 0,06.

\section{RESULTADOS}

En la Tabla 1, se presentan los resultados promedio de los beneficios por sustitución [ver ecuación(1)] y por exportación [ver ecuación (2)], los cuales son calculados para un día de demanda promedio. En todos los casos, los beneficios obtenidos son producto de un proceso de simulación, que se realizó con Monte Carlo y utilizando el Software Risk 5.5. Para las dos empresas en el Esc. 1, se obtienen los mismos beneficios por sustitución $\left(\mathrm{B}_{\mathrm{s}}\right)$ como producto de reemplazar las compras de electricidad a la red tradicional por la autogeneración FV; esto ocurre por cuanto la generación de 300 paneles FV es inferior a la demanda en las dos empresas.

En contraposición, en el Esc.2., en donde se cuenta con 1500 paneles FV, la empresa industrial obtendría mayores $B_{s}$ mediante la autogeneración, como consecuencia de tener mayor demanda de potencia en las horas diurnas que puede ser cubierta con autogeneración. Sin embargo; en el caso del hipermercado que tiene una demanda de potencia mayor a la de la empresa industrial en el período nocturno (18:00 a 22:00 horas), la demanda que logra sustituir es menor; situación que se podría cambiar si contara con tecnologías de almacenamiento. Los beneficios por venta de excedentes $\left(B_{e}\right)$, son mayores para el hipermercado en el Esc.2, como consecuencia de tener mayores excedentes de autogeneración y menor demanda durante las horas diurnas (ver Tabla 1). 
Tabla 1: Beneficios promedio día

\begin{tabular}{|l|c|c|c|c|}
\cline { 2 - 5 } \multicolumn{1}{c|}{} & \multicolumn{2}{c|}{ Empresa industrial } & \multicolumn{2}{c|}{ Hipermercado } \\
\cline { 2 - 5 } \multicolumn{1}{c|}{} & Esc. 1 & Esc. 2 & Esc. 1 & Esc. 2 \\
\hline $\mathrm{B}_{\mathrm{s}}$ & $149.636,0$ & $715.601,0$ & $149.636,0$ & $478.628,0$ \\
\hline $\mathrm{B}_{\mathrm{e}}$ & - & $17.063,4$ & - & 141.171 \\
\hline Beneficio total & $149.636,0$ & $732.664,4$ & $149.636,0$ & $619.899,0$ \\
\hline
\end{tabular}

La Tabla 2 presenta la $Q_{i}$ y los BRD correspondientes al período pico, para las dos empresas y los dos escenarios. Los beneficios promedio, si participa en un PRD-TOU, se calcularon de acuerdo con los períodos de $D_{p}(9: 00$ a 12:00 y 18:00 a 21:00). En la jornada diurna se utilizó la ecuación (7); sin embargo, como se puede observar en las Figuras 1 a 4, en las horas de la noche no se cuenta con autogeneración y, por este motivo, en este período para el cálculo de Qi, se utiliza la ecuación (5). Recordemos que la AGT es igual para las dos empresas, pero la diferencia existente en la cantidad de potencia demanda en $D_{p}$, ocasiona que los $\mathrm{B}_{\mathrm{RDv}}$ sean mayores en el Esc.2, para la empresa industrial y los BRDp en el caso del hipermercado.

Tabla 2: Beneficios promedio por RD

\begin{tabular}{|l|c|c|c|c|}
\cline { 2 - 5 } \multicolumn{1}{c|}{} & \multicolumn{2}{c|}{ Empresa industrial } & \multicolumn{2}{c|}{ Hipermercado } \\
\cline { 2 - 5 } \multicolumn{1}{c|}{} & Esc. 1 & Esc. 2 & Esc. 1 & Esc. 2 \\
\hline $\mathrm{Q}_{\mathrm{i}}$ (9:00 a 12:00 hrs.) kWh & 128,7 & 128,7 & 26,0 & 26,0 \\
\hline $\mathrm{Qi}_{\mathrm{i}}(18: 00$ a $21: 00 \mathrm{hrs})$ kWh & - & & 42,3 & 42,3 \\
\hline $\mathrm{B}_{\mathrm{RDv}}$ & $103.777,5$ & $436.750,3$ & $88.403,0$ & $288.803,8$ \\
\hline BRDp & $103.723,5$ & $30.569,2$ & $82.173,3$ & $199.005,1$ \\
\hline Total Beneficios & $207.629,7$ & $467.448,2$ & $170.644,6$ & $487.877,2$ \\
\hline
\end{tabular}

La Figura 5 presenta los beneficios que se obtuvieron en cada escenario para las dos empresas. La empresa industrial obtiene mayores beneficios, cuando la $A G_{T}>D_{p}$ (sin incluir PRD). Sin embargo, con la inclusión de PRD, es el hipermercado el que obtiene mayores beneficios. Lo anterior se debe a que, mientras los $B_{s}$ se contabilizan con un $\mathrm{PCl}$ que no se modifica a través del período, los Bro tienen dos tipos de tarifas, en las que el período de $D_{v}$ cuenta con una tarifa valle que es menor al precio de compra de las empresas (PCl) y, el costo que se supone se paga por disminución de la demanda IRD, también es menor al PCl.

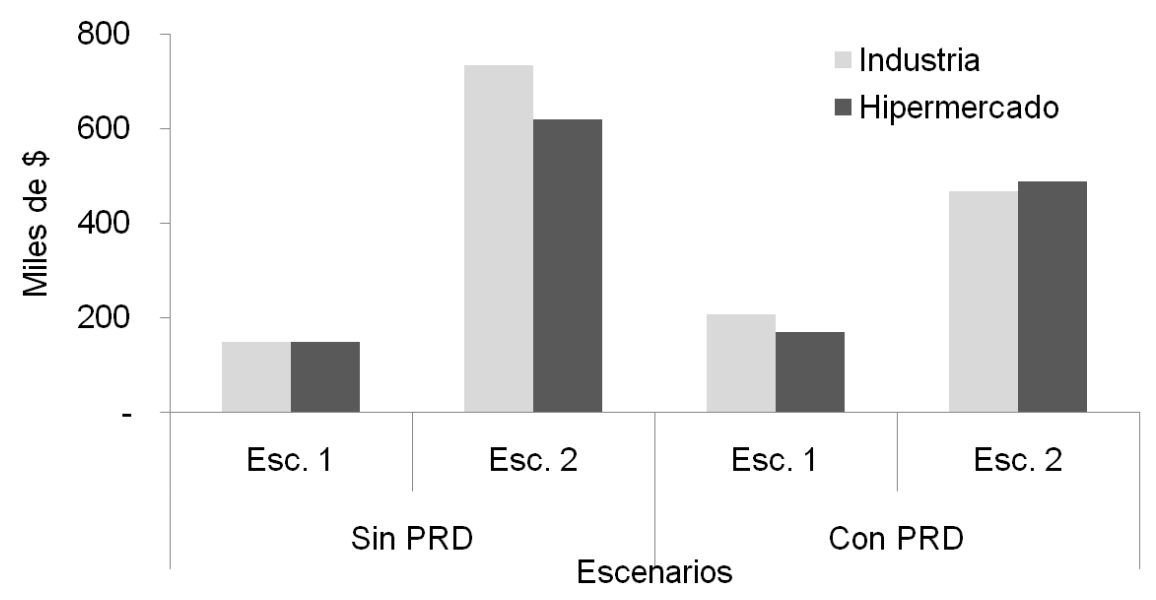

Fig. 5: Beneficios totales.

Bajo los supuestos que se plantean para el caso de los PRD, se generan mayores beneficios al cubrir simplemente el consumo con autogeneración y exportar los excedentes, a participar en PRD. Pero, hay que tener presente que las tarifas para PRD se determinaron con base en el PCl, situación que, en la práctica, puede ser diferente.

A la fecha de elaboración de este trabajo, no se tiene conocimiento de trabajos previos (de otros autores) desarrollados con el enfoque aquí propuesto en el actual marco normativo colombiano. Trabajos que han 
evaluado las FER en otros países, como es el caso de: Zhang, et al. (2014); Ritzenhofen y Spinler (2016) y, Genoese et al. (2016); entre otros, muestran: 1) resultados económicos positivos de las diferentes políticas tendientes a incentivar la inversión en FER, 2) la importancia de considerar la incertidumbre de las FER en el proceso de cálculos de los beneficios económicos y, 3) la flexibilidad que aporta a la gestión del sistema los programas PRD.

\section{DISCUSIÓN FINAL}

A partir de un modelo de gestión de la demanda que considera la incertidumbre de las tecnologías FV y, de los procesos de cálculo de los beneficios económicos que puede obtener un autogenerador, como producto de la gestión de los flujos de potencia en el marco de la reglamentación colombiana, se calculan tres tipos de beneficios: sustitución, exportación y participación en PRD. En cuanto a la sustitución de la demanda, éste es el que más réditos económicos representa, debido al volumen de potencia que se puede cubrir y, el precio con que se contabilizan (los precios del mercado a largo plazo son mayores a los otros precios de referencia); además, su gestión no implica costos adicionales por transmisión al Sistema Interconectado Nacional, ni pagos por gastos de representación a intermediarios, que sí se contabilizarían en el caso de exportación de potencia. La exportación, si bien, es una opción que puede representar beneficios económicos, es la que menos produce por los costos adicionales (pagos de: transporte y de intermediación), y sería necesario, en futuros trabajos, compararlos frente a los costos que representa contar con tecnologías de almacenamiento de potencia, ya que posiblemente sería más beneficioso utilizar los excedentes en horas en que el sistema FER no genera. Como se mencionó, inyectar potencia a la red implica costos adicionales que se pagan a los intermediarios lo que disminuye los beneficios; además, los agentes del mercado en el proceso de establecimiento de las condiciones, no negociarán un precio del kWh mayor al que se paga en el mercado eléctrico colombiano, y seguramente buscarán un precio menor, dada su condición de dominancia.

Se demuestra que la participación en PRD-TOU, permite obtener importantes ingresos en empresas de diferentes sectores económicos y con diferentes curvas de carga. Los PRD, además de ser una novedad en el mercado eléctrico colombiano, pueden llegar a convertirse en uno de los mayores incentivos, si su reglamentación fuese eficientemente planificada por las agencias del estado e implementada por los agentes del mercado eléctrico; éstos, pueden darle el impulso que necesitan las políticas y beneficios tributarios de la Ley 1715; además teniendo en cuenta que en Colombia a diferencia de otros países, la autogeneración con FER no es subsidiada ni asegurada su compra por parte del estado. Los dos casos de estudio que sirvieron de referencia, representan la mayoría de empresas colombianas y bajo los supuestos establecidos, se determinaron en buena medida los beneficios económicos que se pueden obtener con las tecnologías FV y Ios PRD. Lo anterior es un indicativo de la posible viabilidad de este tipo de proyectos y programas. Por el desconocimiento en Colombia de las tecnologías FV y los PRD, se necesitan modelos como los aquí presentados, que permiten determinar, desde el punto de vista económico, la conveniencia de su implementación.

Colombia es un país en el que la regulación sobre autogeneración y PRD es nueva, en la realización de este trabajo, identificamos vacíos legales, que dificultan la puesta en marcha de los lineamientos establecidos en la Ley 1715. A la fecha de elaboración del presente trabajo, no se conoce la reglamentación del sector que establezca los parámetros específicos para la puesta en marcha de PRD, como: tipos de programas a implementar, precios de la electricidad, mercado objetivo de los programas, porcentaje del consumo que se puede transar, condiciones generales de implementación, etc. Lo anterior debe ser determinado, con el fin de garantizar las condiciones para agentes del mercado y consumidores.

\section{CONCLUSIONES}

De acuerdo a los resultados presentados y discutidos en las secciones anteriores, se puede obtener las siguientes conclusiones principales:

1.- Con el Modelo de Gestión de la Demanda elaborado en el marco de la reglamentación colombiana, se identifican básicamente tres tipos de beneficios económicos que aportan valor a este modelo de proyectos: sustitución, exportación y participación en PRD. El modelo es replicable en otros países ajustando los incentivos en cada caso.

2.- De acuerdo con las tarifas del servicio de electricidad que pagan los consumidores (del sector industrial y comercial), la sustitución de la demanda con autogeneración produce mayores beneficios que los PRD o la exportación de excedentes a la red.

3.-Los PRD y los proyectos de autogeneración, bajo los supuestos aquí presentados, generan beneficios económicos, indicativo que sirve de referencia para determinar la contribución de la regulación en el sector eléctrico colombiano y la viabilidad de este tipo de proyectos. 


\section{REFERENCIAS}

Aalami, H., Parsa, M., y Yousefi, G. Evaluation of nonlinear models for time-based rates demand response programs, doi:10.1016/j.ijepes.2014.10.021, Electrical Power and Energy Systems, 65, 282-290 (2015)

Abdi, H., Dehnavi, E., y Mohammadi, F. Dynamic Economic Dispatch Problem Integrated With Demand Response (DEDDR) Considering Non-Linear Responsive Load Models, doi:10.1109/TSG.2015.2508779, IEEE Transactions on Smart Grid, 7(6), 2586-2595 (2015)

Bahrami, S., y Sheikhi, A. An Optimal Feed-In-Tariff Policy for Renewable Energies Using a Markov Model, American Journal of Renewable and Sustainable, 1(1), 1-8 (2015)

Baratto-Callejas, P. Implementación de un programa de respuesta de la demanda de Energía Eléctrica en un Mercado de Clientes No Regulados en Colombia. https://goo.gl/CqkcVC, Rev. Maest. Derecho Econ. Bogotá (Colombia), 6 (6), 259-292, enero-diciembre (2010)

CREG, Regulación Actividad de Autogeneración: Documento CREG-097. (En la web: https://goo.gl/KAFchw, acceso: 18 de Diciembre de 2016), Comisión de Regulación de Energía y Gas (2014a)

CREG, Respuesta a la Demanda, RD, Para el Mercado Diario en Condiciones de Escasez: Documento CREG 056. (En la web: https://goo.gl/DH188y, acceso:18 de Diciembre de 2016), Comisión de Regulación de Energía y Gas (2014b)

DOE, National Action Plan on Demand Response (en la web: https://goo.gl/PXkktt, acceso: 17 de Junio de 2016), The Federal Energy Regulatory Commision Staff, Docket No. AD09-10, June 17 (2010)

Dong, J., Xue, G., y Li, R. Demand response in China: Regulations, pilot projects and recommendations - A review, doi.org/10.1016/j.rser.2015.12.130, Renewable and Sustainable Energy Reviews, 59, 13-27 (2016)

EIA. Independent Statics y Analysis U.S. (En la web: https://goo.gl/szUX5u, acceso: 20 de junio de 2016), Energy Information Administration (2016)

Genoese, M., Slednev, V., y Fichtner, W. Analysis of drivers affecting the use of market premium for renewables in Germany, doi:10.1016/j.enpol.2016.07.043, Energy Policy, 97, 494-506 (2016)

Hopper, N., Goldman, C., y Neenan, B. Demand Response from Day-Ahead Hourly Pricing for Large Customers, doi:10.1016/j.tej.2006.02.002, The Electricity Journal, 19(3), 52-63 (2006)

ISAGEN. Consumir igual y Pagar menos. (En la web: https://goo.gl/oXkk4d, acceso: 7 de julio de 2016). Respuesta Total, 44 (2005)

Mohsenian-Rad, A.H., Wong, V.W., y Jatskevich, J. Autonomous Demand-Side Management Based on GameTheoretic Energy Consumption Scheduling for the Future Smart Grid, doi:10.1109/TSG.2010.2089069, IEEE Transactions on Smart Grid, 1(3), 320-331 (2010)

Muñoz, A., Sáchez, E.F., Cruz, A., y Marín, J. Short-term Forecasting in Power Systems: A Guided Tour, doi:10.1007/978-3-642-12686-4_5, Handbook of Power Systems II, Energy Systems, Heidelberg: Springer, pp. 129-160, Berlin, Germany (2010)

NASA. Atmospheric Science Data Center. (En la web: https://goo.gl/XthBNd, acceso: 17 de Abril de 2015), Atmospheric Science Data Center (2015)

Povh, M., Golob, R., y Fleten, S.E. Modelling the Structure of Long-Term Electricity Forward Prices at Nord Pool, Handbook of Power Systems II, Energy Systems, doi:10.1007/978-3-642-12686-4_7, Heidelberg: Springer, pp. 189-212, Berlin (2010)

Restrepo, A., Manotas, D., y Lozano, C. Portafolio para Autogeneración de Electricidad con Fuentes Renovables en Edificios Comerciales, doi:10.4067/S0718-07642016000100011, Información Tecnológica, 27(1), 91-104 (2016a)

Restrepo, A., Manotas, D., y Lozano, C. Self-generation of Electricity, Assessment and Optimization Under the New Support Schemes in Colombia, doi:10.1109/TLA.2016.7459614, IEEE LATIN AMERICA TRANSACTIONS, 14(3), 1308-1314 (2016b) 
Ritzenhofen, I., y Spinler, S. Optimal desing off fed-in tariffs to simulate renewable energy investments under regulatory uncertaninty-A real options analysis, doi:10.1016/j.eneco.2014.12.008, Energy Economics, 63, 7689 (2016)

Siano, P. Demand response and smart grids-A survey, doi:10.1016/j.rser.2013.10.022, Renewable and Sustainable Energy Reviews, 30, 461-478 (2014)

UPME. Generador de Consultas del Mercado Eléctrico. En la web: https://goo.gl/mYjzR1, acceso: 14 de julio (2016)

UPME-IDEAM. Atlas de Radiación Solar de Colombia, Ministerio de Minas y Energía-Colombia. Bogotá, Colombia (2005)

Wang, Z., Paranjape, R., Sadanand, A., y Chen, Z. Residential demand response: An overview of recent simulation and modeling applications, doi:10.1109/CCECE.2013.6567828, Electrical and Computer Engineering (CCECE), 26th Annual IEEE Canadian Conference, 1-6, Regina, SK, Canada, 5 a 8 de Mayo (2013)

XM S.A. E.S.P. Informe de Precios y Transacciones. (En la web: https://goo.gl/hcnDcx, acceso 18 de Diciembre de 2015), Bogotá: XM (2015)

Zhang, M., Zhou, D., y Zhou, P. A real option model for renewable energy policy evaluation with application to solar PV power generation in China, doi:10.1016/j.rser.2014.08.021, Renewable and Sustainable Energy Reviews, 40, 944-955 (2014)

Zhang, S., Jiao, Y., y Chen, W. Demand-side management (DSM) in the context of China's on going power sector reform, doi:10.1016/j.enpol.2016.09.057, Energy Policy, 100, 1-8 (2017)

Zhou, R., Li, Z., Wu, C., y Chen, M. Demand Response in Smart Grids: A Randomized Auction Approach, doi:10.1109/JSAC.2015.2481208 IEEE Journal on Selected Areas in Communicatios, 33(12), 2540-2564 (2015) 
